\title{
Transition from viscous fingers to compact displacement during unstable drainage in porous media
}

\author{
Fredrik K. Eriksen $\odot,{ }^{1, *}$ Marcel Moura $\odot,{ }^{1, \dagger}$ Mihailo Jankov, ${ }^{1}$ \\ Antoine L. Turquet, ${ }^{1,2}$ and Knut J. Måløy ${ }^{1,3}$ \\ ${ }^{1}$ PoreLab, the Njord Center, Department of Physics, University of Oslo, \\ P. O. Box 1074 Blindern, 0316 Oslo, Norway \\ ${ }^{2}$ NORSAR, 2007 Kjeller, Norway \\ ${ }^{3}$ PoreLab, Department of Geoscience and Petroleum, Norwegian University of Science and Technology, \\ 7031 Trondheim, Norway
}

(Received 5 August 2021; accepted 30 November 2021; published 14 January 2022)

\begin{abstract}
We describe a crossover from the viscous fingering instability to a compact invasion regime during viscously unstable drainage of porous media, and we investigate the underlying mechanisms of this compact fluid displacement. The study is based on a series of drainage experiments in a radial porous Hele-Shaw cell where we systematically vary the viscosity of the defending (wetting) fluid and the overpressure of the invading (nonwetting) fluid to map out the resulting invasion structures as a function of viscosity ratio and injection pressure. We show that above a threshold of injection pressure and viscosity ratio a more stable and compact invasion structure emerges within the viscous fingering patterns, i.e., a roughly circular displacement with viscous fingers on the outside. The onset of the stable displacement is found to begin at a rather low viscosity ratio $M$ between the invading and defending fluids, i.e., when $M \geqslant 10^{-3}$ for injection pressures of 3-5 $\mathrm{kPa}$. We find that the ratio between the length of the outer fingers and the size of the compact invasion scales with the viscosity ratio and approaches a more or less constant value during growth, resulting in structures with proportionate growth and larger compact invasions for higher viscosity ratios. As opposed to the viscous fingering instability, we describe rich ganglion dynamics within the compact invasion structures and show that the pressure gradient is not screened by the outer fingers.
\end{abstract}

DOI: 10.1103/PhysRevFluids.7.013901

\section{INTRODUCTION}

Two-phase flow in porous media is of central importance in a wide range of fields from fundamental research to applied areas and practical daily life problems like making a cup of tea. It is clearly a multidisciplinary subject and its complex nature has been studied by hydrologists, physicists, chemists, geoscientists, biologists, and engineers for decades. The structures observed are controlled by the characteristic forces involved like viscous [1-4], capillary [4-6], and

\footnotetext{
*f.k.eriksen@fys.uio.no

†marcel.moura@fys.uio.no
}

Published by the American Physical Society under the terms of the Creative Commons Attribution 4.0 International license. Further distribution of this work must maintain attribution to the author(s) and the published article's title, journal citation, and DOI. 
gravitational forces [7] in addition to wetting properties [6,8] and fluctuations in the local geometry of the porous medium. These structures range from compact to the ramified and fractal $[9,10]$.

When a nonwetting fluid invades another wetting fluid at low injection rates, capillary forces will dominate the viscous forces and a capillary fingering regime $[5,11]$ is observed. The structure in this regime is well described by invasion percolation with trapping [5,12-16]. At high injection rates, however, the patterns strongly depend on the viscosities of the two fluids. When the viscosity of the displaced fluid $\mu_{d}$ is higher than the viscosity of the invading fluid $\mu_{i}$, a viscous fingering instability is observed [17], creating a fractal ramified structure [2,3]. However, when the injected fluid has a higher viscosity than the displaced fluid a compact stable structure is formed. A detailed phase diagram for two-phase flow was developed by Lenormand et al. [11] performing quasi-twodimensional (2D) experiments and simulations, which also predicted the boundary between compact growth and viscous fingering. The transition between viscous and capillary fingering has been studied both experimentally $[4,11,18]$ and by computer simulations $[11,18,19]$. Simulations [20] and experiments [21] have also been used to investigate the transition between capillary fingering and compact growth. However, few studies have been performed on the transition from viscous fingering to compact displacement [22,23], which is the main purpose of this study. Ferer et al. [22,23] performed simulations where they predicted the crossover length of the clusters from fractal viscous fingers. Those simulations were performed with zero surface tension. Our study is an experimental investigation of this transition where we observe the coexistence of external fingers and a compact growth with rich internal cluster dynamics. The most unstable wavelength given by the theory by Saffman and Taylor [17] is governed by the competition between viscous and capillary forces and predicts the onset of instability but does not predict the coexisting growth of a compact internal zone and viscous fingers. This compact growth is observed in our experiments even when the viscosity of the displaced fluid is 1000 times higher than that of the invading fluid.

The observed viscous fingering structures resemble the fractal structures found in other irreversible growth processes like electrolytic deposition [24,25], dielectric breakdown [26], dissolution of porous materials [27], and diffusion limited aggregation (DLA) [28]. The analogy between viscous fingering and DLA and between compact displacement and anti-DLA was first pointed out by Paterson [29]. The analogy that exists between these processes is built on the fact that in the continuum description both processes are described with a Laplace equation with the same boundary conditions.

Bischofberger et al. [30] studied the fingering instability in a Hele-Shaw cell by using two miscible fluids. They found three different regimes characterized by the viscosity ratio $\mu_{i} / \mu_{d}$. When $\mu_{i} / \mu_{d}$ increases they observe a transition from fractal viscous fingering with an internal growing compact zone to a situation with a growing compact structure with blunt external features without tip splitting. Finally, for $\mu_{i} / \mu_{d}>0.3$ they observed a stable regime without any external fingers. However, there are two important differences between [30] and our study. In their case the fluids are miscible with zero surface tension and no porous medium, while in our experiments a finite surface tension and a porous medium are present.

A remarkable feature which is often observed in biological growth processes, but which is hard to find in physical systems outside biology was observed in our experiments: the fact that the structures generated look similar when rescaling with the size of the total structure. A good example is a fish that looks nearly similar independent of the age. This feature, called proportionate growth, keeps the structure self similar by rescaling with the size of the structure. In the transition from stable growth to viscous fingering, apart from an initial regime, we observed proportionate growth. This type of growth was also observed in the experiments by Bischofberger et al. [30] in the regime with external blunt structure without tip splitting. We further found that the ratio between the characteristic length scale of the length of the external fingers and the compact growth depends on the viscosity ratio $\mu_{i} / \mu_{d}$ for a fixed inlet pressure.

Furthermore, we have observed a rich dynamical behavior inside the compact structure with coalescence, fragmentation and motion of the trapped air clusters. The intense ganglion dynamics [31] observed reminds us about what is seen in the steady-state regime with a simultaneous injection 


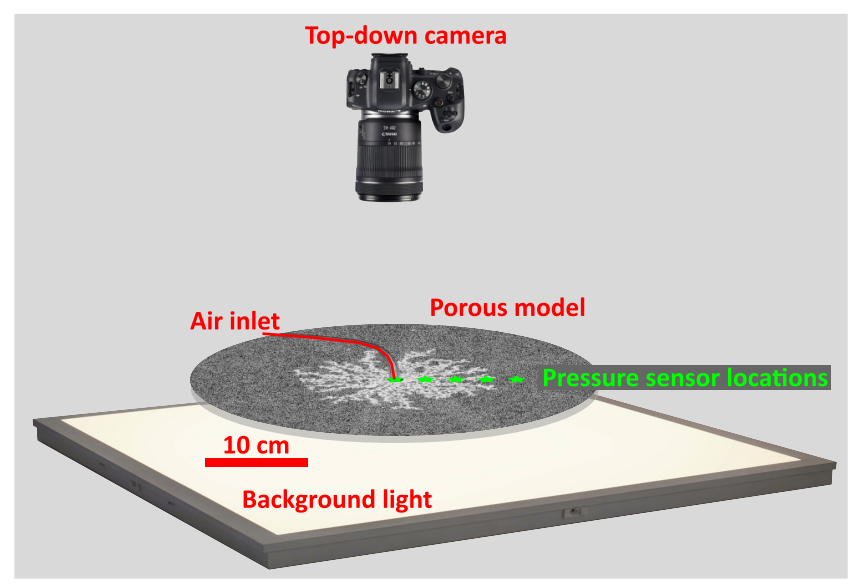

FIG. 1. The porous model is a circular Hele-Shaw cell (20-cm radius) containing a disordered monolayer of 1-mm glass beads at fixed positions. The fluid inlet is at the center of the model and the outlet is the unsealed outer perimeter of the model. The model is initially saturated with a viscous liquid (dark) while air (white) is injected with a constant overpressure during the invasion experiment. The experiments are recorded with a camera facing top-down above the cell and a uniform white background light is transmitted through the model to provide good contrast between the defending and invading fluids. The green stars indicate the locations of the pressure sensors in the pressure recording experiments.

of two fluids [32-34]. To quantify the ganglion dynamics we have developed a technique that measures the number of times one position in space flips its state, from being occupied by one fluid to the other. This flipping matrix technique turns out to be an efficient way to quantify the activity level of the cluster dynamics in the compact zone and other flow regimes with high cluster activity like the steady-state regime.

\section{METHODOLOGY}

\section{A. Experimental setup}

The flow cell used in our experiments is a circular and horizontal Hele-Shaw cell containing a quasi-2D porous medium with a $20-\mathrm{cm}$ radius. See Fig. 1 for an illustration of the experimental setup. The flow cell has a fluid inlet in the center, while the fluid outlet is the unsealed outer perimeter of the model (open to the atmosphere). The porous model is formed by a disordered monolayer of glass beads with diameters in the range $1.00 \pm 0.10 \mathrm{~mm}$. This monolayer is confined inside the Hele-Shaw cell between a thick glass plate on the top and a pressure cushion on the bottom, with a $250 \mathrm{cmH}_{2} \mathrm{O}$ confining pressure $\left(1 \mathrm{cmH}_{2} \mathrm{O} \approx 100 \mathrm{~Pa}\right)$. The beads are glued between two plastic films and pressed toward the glass disk by the pressure cushion. This setup makes the porous medium rigid, i.e., the fluid motion does not interfere with the pore network. We use the same type of setup as in e.g., [4] where a detailed description of the flow cell is given. During an experiment, we initially saturate the porous medium with a wetting liquid, before air (nonwetting) is injected into the cell at a constant overpressure. The resulting fluid displacement is recorded with a Canon EOS R digital camera, placed directly above the cell, at a frame rate of 25 images/s and a spatial resolution of $2160 \times 3840$ pixels $(1$ pixel $\approx 0.2 \mathrm{~mm}$ ). The duration of an experiment is in the range of $1 \mathrm{~s}$ to $2.5 \mathrm{~min}$, depending on the overpressure of the invading air and the viscosity of the defending fluid. We conducted a set of 23 experiments, where we systematically varied the viscosity of the defending fluid and the overpressure of the invading fluid. In addition to the systematic experiments, we conducted specific experiments to measure the evolution of pore pressure during the flow. In these experiments we replace the top-plate of the Hele-Shaw cell with one that has five Honeywell 


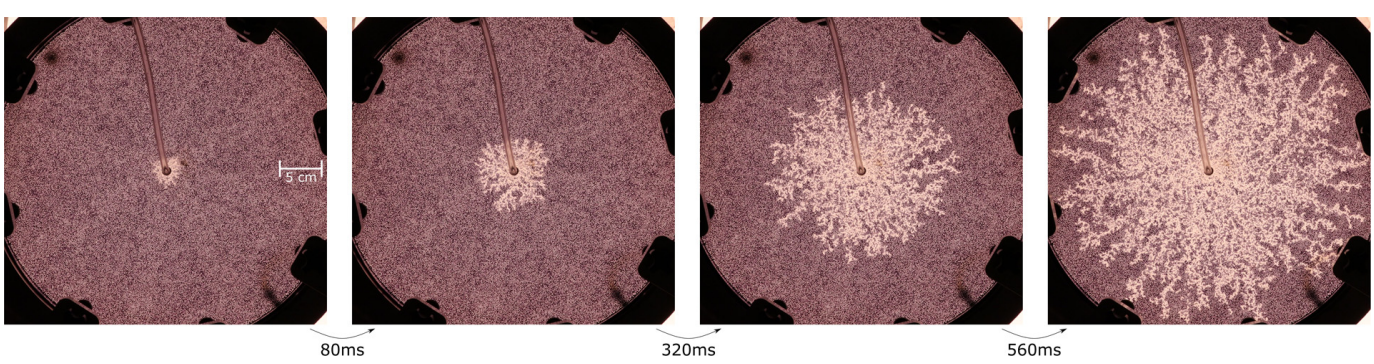

FIG. 2. Four snapshots illustrating the temporal evolution of a typical experiment in which the defending fluid was a water-glycerol mixture with glycerol concentration $C_{g}=20 \%$ (by mass) and the air pressure was $50 \mathrm{cmH}_{2} \mathrm{O}$. The time delay between consecutive snapshots is shown in the figure.

26PCAFG6G pressure sensors placed along a line radiating from the injection center (indicated by green stars in Fig. 1). The first sensor is placed at the inlet, the others are placed at the radii of 3, 6, 9, and $12 \mathrm{~cm}$. The pressure signals are recorded during the experiments with a NI-DAQ USB-6212 data acquisition card at a sampling rate of $5 \mathrm{kHz}$. The pressure signal is smoothed to reduce electronic noise with a $20 \mathrm{~ms}$ moving average window, i.e., averaging around each data point with 50 samples before and after.

We inject air into the model from a pressurized air tank, which is connected to a water overflow system that maintains the overpressure. We have conducted experiments with air pressures of 10 , 30 , and $50 \mathrm{cmH}_{2} \mathrm{O}$. Figure 2 shows the temporal evolution of a typical experiment with invading air pressure of $50 \mathrm{cmH}_{2} \mathrm{O}$. The defending fluid in this case was a water-glycerol mixture with glycerol concentration $C_{g}=20 \%$ (by mass), see Table I.

\section{B. Defending fluids}

We have used 10 different viscosities for the defending fluids, which are listed in Table I. The viscosity range is from 0.995 to $217 \mathrm{mPa} \mathrm{s}$, achieved by mixing glycerol concentrations in type II water from $0 \%$ to $90 \%$ by mass, respectively. The water was dyed with nigrosine (1.1 g nigrosine per $1000 \mathrm{~g}$ water) to give the defending fluid a dark color in contrast to the white color of the invading air. The viscosities are calculated using an empirical formula with an error of $<5 \%$ [35] by inserting the laboratory temperature of $20.4 \pm 0.5^{\circ} \mathrm{C}$ that was recorded over the course of the experiments. The liquid-air surface tension is given as the tabulated values at room temperature [36] in Table I

TABLE I. Viscosities estimated from the glycerol concentration in water for the 10 defending liquids at the laboratory temperature of $20.4 \pm 0.5^{\circ} \mathrm{C}$ and tabulated surface tension values at room temperature. $C_{g}$ is the glycerol concentration in the mixture, $\mu_{d}$ is the liquid viscosity, and $\gamma$ is the liquid-air surface tension.

\begin{tabular}{lcc}
\hline \hline$C_{g}(\%$ by mass $)$ & $\mu_{d}(\mathrm{mPas})$ & $\gamma(\mathrm{mN} / \mathrm{m})$ \\
\hline 0 & $0.995 \pm 0.012$ & 72.0 \\
10 & $1.284 \pm 0.016$ & 70.5 \\
20 & $1.722 \pm 0.024$ & 69.5 \\
30 & $2.43 \pm 0.04$ & 68.5 \\
40 & $3.64 \pm 0.06$ & 67.9 \\
50 & $5.91 \pm 0.11$ & 67.4 \\
60 & $10.72 \pm 0.23$ & 66.9 \\
70 & $22.6 \pm 0.6$ & 66.5 \\
80 & $59.4 \pm 1.8$ & 65.7 \\
90 & $217 \pm 8$ & 64.5 \\
\hline \hline
\end{tabular}


(a)

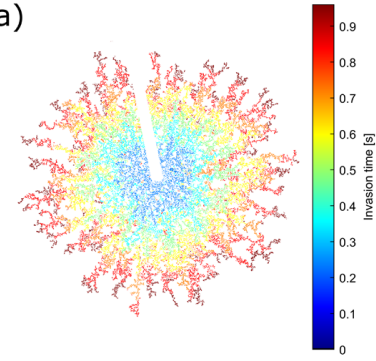

(b)

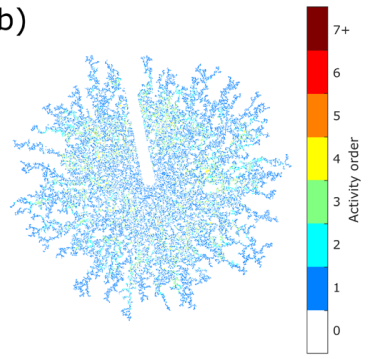

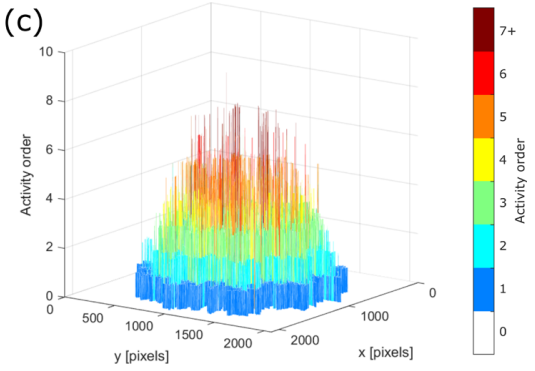

FIG. 3. (a) Invasion matrix for the experiment shown in Fig. 2. The color code indicates the time in seconds at which a given point is first reached by the air phase (see video in the supplementary material S1 [37]). (b) Flipping matrix for the same experiment. The color code indicates the number of times that a given pixel has flipped between liquid-filled and air-filled states. (c) Activity landscape. This is a 3D plot of the data presented in the flipping matrix in (b). The height corresponds to the activity order of each point (number of state flips).

and is not very sensitive to the glycerol concentration. The surface tension is $72 \mathrm{mN} / \mathrm{m}$ for pure water and decreasing about $10 \%$ to $64.5 \mathrm{mN} / \mathrm{m}$ for $90 \%$ glycerol. The liquid-air contact angles $\alpha$ were measured with the sessile drop technique on glass and plastic film substrates with a Krüss DSA25E drop shape analyzer and showed that they remain constant within the $5^{\circ}$ measurement uncertainty for all the liquids: for glass $\alpha=23 \pm 4^{\circ}$ and for plastic $\alpha=75 \pm 4^{\circ}$. This indicates that the invasion structures we observe are not impacted by significant differences in wetting properties between our defending liquids.

\section{The invading matrix and flipping matrix concepts}

The set of experiments with different defending phase viscosities and invading phase pressures have yielded a wide range of dynamical features. The observed invasion dynamics included the standard viscous fingering behavior in porous media [2,3,38,39], particularly in the regime of higher defending fluid viscosity, but we have also observed many additional features that are unaccounted for in the literature. In order to better visualize and understand these features, we will introduce two matricial concepts: the invading matrix and flipping matrix.

The invading matrix is a spatiotemporal map of the invasion pattern [40,41]. It measures the time at which a given location in the pore space has been first reached by the invading phase. In a simple primary drainage scenario, in which a nonwetting phase enters a porous network saturated with a wetting phase, this matrix encodes the full temporal information of the invasion dynamics [15]. In Fig. 3(a) we show the invading matrix for an experiment where the glycerol concentration was $C_{g}=20 \%$ and the invading pressure was $50 \mathrm{cmH}_{2} \mathrm{O}$ (same experiment depicted in Fig. 2). The whole experiment lasted less than $1 \mathrm{~s}$, see colorbar (a video of the invading matrix evolution for this experiment is available in the supplementary material S1 in Ref. [37]). In Fig. 3(a) we notice that the fingering is much more pronounced closer to the rim of the image. In the central part, the front advanced in a much more compact manner. We will get back to this point later.

The standard viscous fingering behavior corresponds to a fast primary drainage scenario, i.e., once a pore is invaded by the nonwetting phase, it is never invaded again by the wetting phase. In our experiments we have observed that many experiments deviate from this standard scenario: A rich ganglion dynamics is observed in which drainage and imbibition alternate as pores are invaded by both phases in a rapid succession. This behavior is not captured by the invading matrix, which, as previously mentioned, only measures the time of first air invasion of a given point. In order to quantify the ganglion activity, we have introduced the concept of the flipping matrix, which we explain next. 
Simply put, the flipping matrix quantifies the number of times a given pixel in the pore space has its state flipped from liquid filled to air filled and vice versa. We call this number the activity order. An undisturbed region of the network, i.e., an area that was never reached by the air phase, has activity order 0 . A region that was reached by the air phase but that did not see any other invasion activity until the end of the experiment has activity order 1 . A region that was reached by air but was again filled by liquid before the end of the experiment has activity order 2, and so on. While the invading matrix shows the time of first invasion of the network, the flipping matrix shows the corresponding activity order. A standard primary drainage experiment has only $0 \mathrm{~s}$ and $1 \mathrm{~s}$ in the flipping matrix. Any experiment in which ganglion dynamics is present has higher-order values (larger than 1) in the flipping matrix. In the supplementary material S2 [37] we show a video where we zoom in on different parts of the model to show the local flipping between the air-filled and liquid-filled states.

Figure 3(b) shows the flipping matrix corresponding to the same experiment as in Fig. 3(a). Notice the presence of higher-order values, indicating strong ganglion activity. In the next section we will explain how we can use the flipping matrix to produce a global quantification of the ganglion activity intensity and we will define two global measures of ganglion dynamics intensity. But before moving into that, let us consider a different representation of the flipping matrix, which will prove useful later. In Fig. 3(c) we present a 3D plot of the flipping matrix, where for each point in the matrix we associate a height corresponding to the activity order of that point (i.e., the number of times the state of the given point has flipped between liquid- and air-filled states). This plot looks like a dome or mountain, so we call it the activity order landscape. Although the data are precisely the same as in the flipping matrix, this representation brings some visual advantages, which will become more clear in the next section.

All image analysis in this paper, including that necessary for the computation of the invading and flipping matrices, was done using MathWorks MATLAB. In the experimental images, the defending liquid phase is seen in a darker color, while the invading air phase and the glass beads forming the porous matrix appear as a much lighter color, see Fig. 2. This sharp difference in color intensity makes it simple to segment the images to isolate the growing air phase. In order to perform this segmentation, we initially subtract the initial frame from all subsequent frames. After the image subtraction we are left with an image of what has changed between the frames, i.e., the invading air cluster. We apply a color thresholding procedure to turn this image into a binary matrix, with ones in the positions corresponding to the air phase and zeros elsewhere. At this stage we are left with a large sequence of binary images of the invading air cluster, one for each time step. This image sequence is used to compute both the invading matrix and the flipping matrix: For the invading matrix we start with a matrix of zeros and for each time step for which the binarized image shows the invasion of new pores, we associate the respective time to those newly invaded pores. For the flipping matrix, we again start with a matrix of zeros and check the amount of times the index of the binarized image of a given pixel flips from 0 to 1 or from 1 to 0 along the time series. The number of state flips (activity order) is then associated to that particular pixel in the flipping matrix and we repeat the procedure for all pixels.

\section{Global measures of ganglion dynamics intensity}

The flipping matrix and associated activity order landscape [Figs. 3(b) and 3(c)] provide visual representations of the ganglion activity for the whole duration of the experiment. Although these measures give direct visual confirmation of the local ganglion activity, we need to also quantify the global intensity of such activity. This quantification is particularly relevant when we want to compare between different experiments. In order to fulfill that goal we have defined two global ganglion dynamics measures, which we call $\psi_{1}$ and $\psi_{2}$.

Before defining the measures $\psi_{1}$ and $\psi_{2}$, we note that the existence of pixels with activity order higher than 1 is one of the signatures of ganglion dynamics. In a system where only primary drainage occurs, once the state of a given location changes from liquid filled to air filled (transition 0 to 1 in 
the flipping matrix), no further activity occurs on that point. The flipping matrix would show only the deep blue part in Fig. 3(b), corresponding to the widest primary drainage plateau at the bottom of the activity order landscape, see Fig. 3(c). We can then divide the activity order landscape into two regions, the primary drainage plateau having height 1 and a ganglion dynamics region above 1 . This separation makes our definitions of the global ganglion dynamics measures easily understandable.

We define $\psi_{1}$ as the ratio between the volume of the region above the primary drainage plateau in the activity order landscape and the total projected area of the landscape onto the $x y$ plane. If $d A$ is the area of a pixel and $O(i, j)$ is the activity order of pixel $(i, j)$, then this definition reads

$$
\psi_{1}=\frac{\sum_{i, j}[O(i, j)-1] d A}{\sum_{i, j} d A},
$$

where the summation runs over all pixels $(i, j)$ in the invaded region, i.e., all pixels having $O(i, j) \geqslant$ 1. Notice that the denominator corresponds simply to the total invaded area, i.e., the total area of pixels that were invaded at least once by the air phase.

$\psi_{1}$ can be intuitively understood as the global average number of times a pixel invaded by air will have its state flipped again along the experiment. If no ganglion dynamics is present, $\psi_{1}=0$, meaning that once a pixel flips from liquid to air (primary drainage), then it never flips again.

Our second measure $\psi_{2}$ is defined as the ratio between the projected area of the region above the primary drainage plateau and the total projected area of the landscape. This definition differs from $\psi_{1}$ in the numerator: While in $\psi_{1}$ we considered the volume of the region above the primary drainage plateau, here we consider the projected area of that region onto the $x y$ plane. Symbolically it reads

$$
\psi_{2}=\frac{\sum_{i^{\prime}, j^{\prime}} d A}{\sum_{i, j} d A},
$$

where the summation in the numerator runs over all pixels $\left(i^{\prime}, j^{\prime}\right)$ having $O\left(i^{\prime}, j^{\prime}\right) \geqslant 2$ and the one in the denominator runs over pixels $(i, j)$ having $O(i, j) \geqslant 1$ [as in Eq. (1)]. Notice that $\psi_{2}$ completely ignores the order of a given pixel (its height in the activity landscape), all that matters is if a pixel has flipped its state or not after being invaded by air. If it has, then it is counted in the numerator of Eq. (2).

$\psi_{2}$ can be intuitively understood as the average probability that a pixel reached by air will eventually flip its state at least once again. If no ganglion dynamics is present, then $\psi_{2}=0$.

Both measures $\psi_{1}$ and $\psi_{2}$ can be used to quantify ganglion dynamics intensity and, broadly speaking, they are correlated in the sense that generally when an experiment scores high (low) in one measure, it tends to score high (low) in the other. However, one could think of some extreme scenarios in which this correlation could potentially fail. Consider, for example, a porous medium that is mostly homogeneous in all its physical properties, apart from some narrow stripe where the permeability is very different from average. This difference (it could be a fracture, for instance) could locally induce strong ganglion activity with the breakup of the invading phase into a stream of bubbles. Pores belonging to this zone could experience many state flips, which would occur in a high activity order and high $\psi_{1}$ value. However, if the extent of this zone is not too long, then $\psi_{2}$ would not present a very high value. To put it differently, in the case in which just a few pores present very intense ganglion activity, a system could present a high value of $\psi_{1}$ and low value of $\psi_{2}$, so the correlation between the quantities would be lost in such extreme scenarios. We anticipate that for the experiments analyzed here, this loss of correlation was not observed, as no such large heterogeneities are present in the porous samples we used.

Notice that, due to the explicit accounting of the exact number of state flips of all pixels, the quantity $\psi_{1}$ is much more prone to errors than $\psi_{2}$. This is particularly true to pixels belonging to the liquid-air interface, which can sometimes be counted as air filled and sometimes as liquid filled, thus artificially increasing the value of their activity order (number of flips between states). For that reason, it is convenient to have the two separate measures $\psi_{1}$ and $\psi_{2}$ in the analysis of the 


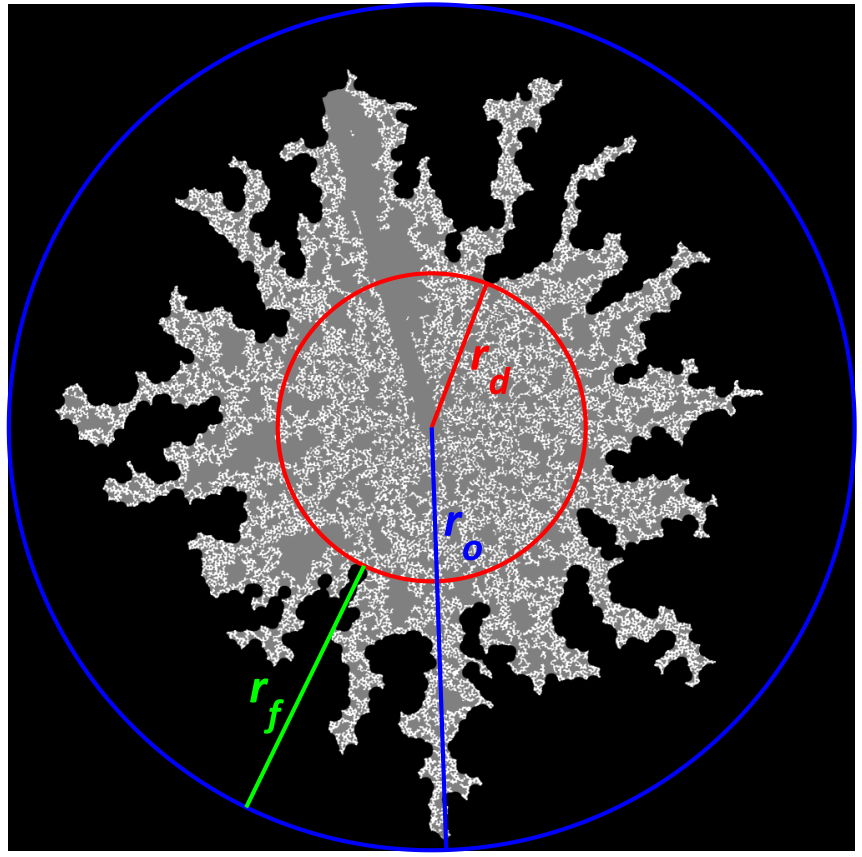

FIG. 4. Illustration of the coarser invaded (gray) and noninvaded (black) regions, with the original invasion structure on top (white). The circles illustrate the obtained length scales: the compact invasion radius $r_{d}$ (red), outer pattern radius $r_{o}$ (blue), and the outer finger length $r_{f}$ (green line).

ganglion dynamics intensity. Reassuringly, as we will see in Sec. III E, our conclusions are the same irrespective of the measure used, as, for the experiments presented, both measures produced similar results. This indicates that the number of pixels that are miscounted in $\psi_{1}$ becomes negligible in comparison with the total number of invaded pixels [that goes in the denominator in the definition of Eq. (1)].

\section{E. Characterizing the radii of the densely invaded region and the outer pattern}

The radii $r_{d}$ of the densely invaded region and $r_{o}$ of the total pattern, as shown in Fig. 4, are estimated by image processing as follows. First, the binary image of the pattern is found by subtracting the actual image with the initial image before invasion and then applying an intensity threshold that returns the air invaded pores as white pixels and the liquid-filled pores, glass beads, and background as black pixels. Note that shadow from the inlet tubing and injection hole results in black pixels even if pores here are invaded. We account for this artifact by filling the shadow region with white pixels up to the radius of the adjacent invasion structure. Next, the binary invasion images are segmented into invaded and noninvaded regions on a coarser scale, removing trapped liquid clusters and fine details. This is done in order to distinguish viscous fingers and compact invasion in a consistent way for all the experiments. The images are segmented into invaded and noninvaded regions by scanning a small neighborhood around each pixel in the image (both black and white) and define the noninvaded region as the union of neighborhoods that does not contain any invaded pixel. To ensure that liquid fjords between close fingers are not counted as trapped clusters, we connect clusters of the noninvaded region that are closer than 1 pore size. Finally, we remove the trapped noninvaded clusters by keeping only the largest noninvaded cluster, i.e., the liquid surrounding the invasion structure. The invaded region is then the rest of the image pixels inside the noninvaded region, including trapped liquid clusters. This method produces a coarse image of the invasion that 


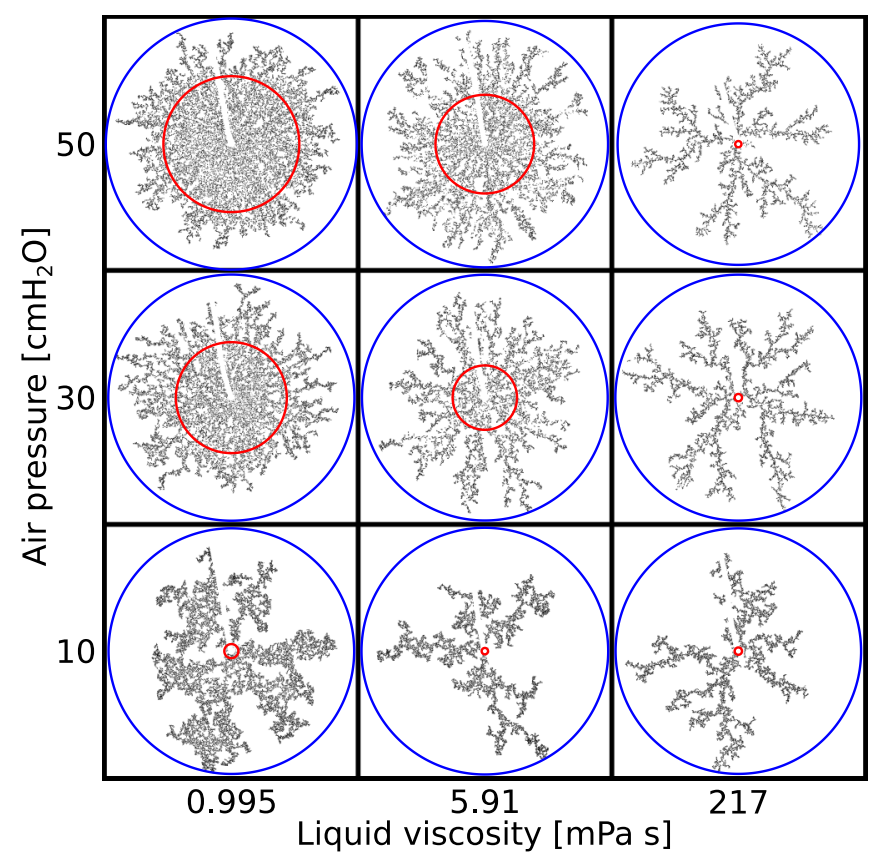

FIG. 5. A viscosity-pressure diagram of the breakthrough patterns. The red circles indicate the radius of the compact invasion zone and the blue circles indicate the total pattern radius. A similar phase diagram for all the experiments is found in the supplementary material S3 in Ref. [37].

completely covers the compact invasion while preserving a coarse shape of the outer fingers as shown in Fig. 4.

The neighborhood we use to characterize the coarse invasion structures is a disk of 3 pore sizes in diameter. This neighborhood size was determined by testing 10 disk sizes $(d=1,2, \ldots, 10$ pore sizes) and comparing the results with manual input of the compact radius for 60 random images ( 3 random images from each experiment). The disk size $d=3 \mathrm{~mm}$ was found to give the best results compared with the manual input from visual inspection, i.e., with $d=3 \mathrm{~mm}$, the algorithm returns $r_{d}$ with a root-mean-square error of $3.5 \mathrm{~mm}$. Three sets of visual inspections gave the manual input of $r_{d}$ a standard deviation of $\pm 2.2 \mathrm{~mm}$. An important point for the choice of neighborhood size is that we optimized the parameter to get output that matches well with what we see visually for all the experiments.

It is straightforward to obtain the outer pattern radius $r_{o}$ from the binary image, we just record it as the length from the injection center to the most advanced white pixel. Then we define the compact invasion radius $r_{d}$ to be the distance from the air inlet to the closest pixel of the noninvaded region of the coarser invasion image (corresponding to the tip of the longest liquid fjord).

\section{RESULTS}

\section{A. Initial observations and characterization of the invasion front}

Figure 5 shows a phase diagram of nine selected experiments, i.e., the breakthrough patterns for $\Delta P=10,30$, and $50 \mathrm{cmH}_{2} \mathrm{O}$, with $C_{g}=0,50$ and $90 \%\left(\mu_{d}=0.995,5.91\right.$, and $217 \mathrm{mPa} \mathrm{s}$, respectively). For the lowest injection pressure $\left(10 \mathrm{cmH}_{2} \mathrm{O}\right)$ we see a crossover from capillary fingers to viscous fingers with increasing viscosity of the defending fluid (from left to right). For the low and middle viscosities $(0.995$ and $5.91 \mathrm{mPa} \mathrm{s})$ we see a transition from capillary/viscous fingers toward more compact invasion patterns with increasing injection pressure (from bottom to 


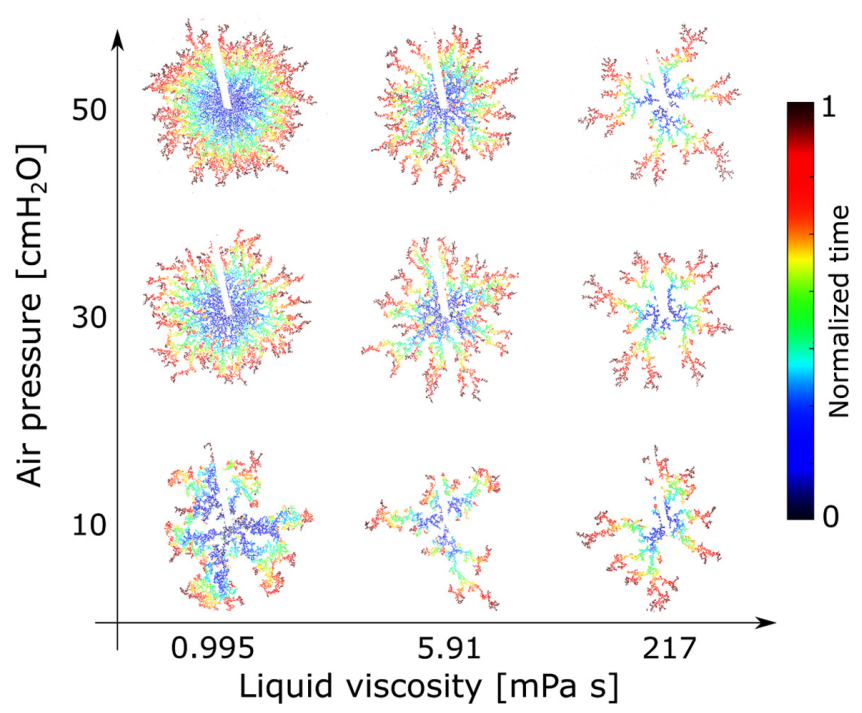

FIG. 6. Invading matrix diagram corresponding to the same experiments shown in Fig. 5. The colormap indicates the normalized time of invasion going from blue (start of the invasion) to red (breakthrough time). A complete version of this diagram with all 23 experiments analyzed is found in the supplementary material S4 in Ref. [37].

top). For the highest viscosity ( $217 \mathrm{mPa}$ s) the patterns remain in the viscous fingering regime at all the injection pressures we used. The red circles indicate the radius of the densely invaded porous media, $r_{d}$, and the blue circles indicate the outer radius of the invasion patterns $r_{o}$. In the experiments without compact invasion, $r_{d} \approx 6 \mathrm{~mm}$ which is the radius of the inlet hole. The diagram in Fig. 5 suggests that there is a transition from viscous fingers (VF) to a compact invasion regime (CI) above an injection pressure threshold, and that this threshold increases with higher fluid viscosity. The onset of the compact invasion appears to begin inside an outer front of viscous fingers, and the ratio of outer finger length over the compact radius $R=\left(r_{o}-r_{d}\right) / r_{d}$ seems to decrease for higher injection pressure and lower defending fluid viscosity. As will be discussed later we also observe that the invasion structures have proportionate growth, i.e., that the structures are self-similar during growth. This is due to the fractal properties in the VF regime, and for CI that the ratio $R$ stabilizes toward a more or less constant value as the structures grow. A larger phase diagram including all the experiments is found in the supplementary material, see S3 in Ref. [37].

\section{B. Spatiotemporal evolution and invasion pattern at breakthrough}

As previously mentioned, we stopped our experiments at breakthrough, i.e., when the air first reaches the rim of the model. The breakthrough time varied by more than 2 orders of magnitude between the experiments, from about $0.8 \mathrm{~s}$ (for the experiment with the lowest liquid viscosity and highest injection pressure) to about $146 \mathrm{~s}$ (experiment with the highest liquid viscosity and lowest injection pressure). This vast difference of experimental durations resulted in an equally vast array of invasion patterns. Figure 6 shows the invading matrix at breakthrough for a set of 9 experiments, with three different liquid viscosities and air pressure values (see numbers in the figure). The colormap indicates the time of first invasion by air of a given pixel, normalized by the breakthrough time (i.e., here the breakthrough time is taken as $t_{b}=1$ for all experiments). A more complete high resolution image with the analysis of all 23 experiments is included in the supplementary material, see S4 in Ref. [37]. 
From the analysis of Fig. 6 we can indeed observe a collection of different invasion patterns. The well-documented viscous fingering regime $[2,3,38,39,42]$ is seen on the right column, where the fingers get thinner and more pronounced for higher values of injecting air pressure. This is expected, as the higher the pressure, the higher the velocities involved which in its turn translate into higher capillary numbers and smaller finger widths $[43,44]$.

On the top left part of the figure we see a completely different regime. Here the defending fluid viscosity is low and the injection pressure is high. We see that close to the center, the invasion happens in a fast sweeping manner, in which air enters the medium in an abrupt motion. This part of the diagram is dominated by inertial effects, and the heterogeneous structure of the pore network is hardly felt by the invading air phase close to the center of the model. It is only closer to the rim of the model that we see the development of fingers, as the inertial effects reduce with distance to the center due to the reduction of local fluid velocity $v$ (which will decay due to the radial geometry of the medium) and consequently of the Reynolds number $\operatorname{Re}=v l / v_{d}$. Here $l$ is a characteristic length scale over which the velocity varies and $v_{d}$ is the kinematic viscosity of the displaced fluid. Looking at Fig. 6 as a whole, we can say that generally speaking the Reynolds number increases as we move toward the top left region (lower viscosities and higher pressures and velocities). Later in Sec. III E we will see that this region of the diagram is also characterized by strong ganglion activity. An accurate computation of the Reynolds number would require local measurements of the velocity fields at the pore scale. Although we have not measured the local pore velocity, an estimate can be given if we consider the velocity of liquid-air interface instead. If we consider the two first snapshots in Fig. 2, then we see that the front has grown by approximately $2.3 \mathrm{~cm}$ during the $80-\mathrm{ms}$ time interval between the pictures. Using an estimate of the local fluid velocity as $v=2.3 \mathrm{~cm} / 80 \mathrm{~ms}=0.28 \mathrm{~m} / \mathrm{s}$, we compute the Reynolds number to be approximately $\operatorname{Re} \approx 177$ if we use the typical pore size as the appropriate length scale in the Reynolds number formula $(l=1 \mathrm{~mm})$. If one instead uses the system radius as the appropriate length scale $(l=20 \mathrm{~cm})$, and the duration of the experiment as the appropriate timescale, then the Reynolds number becomes approximately $\operatorname{Re} \approx 24000$, where the velocity was computed as $v=20 \mathrm{~cm} / 1 \mathrm{~s}=0.2 \mathrm{~m} / \mathrm{s}$, see Fig. 2. Irrespective of the specific Reynolds number definition used, the numbers are far above the domain where the flow is purely laminar, so inertial effects are bound to be present, particularly in the experiments in the upper left corner of Fig. 6.

\section{Pattern growth and scaling}

Figure 7 shows the ratio

$$
R=\frac{r_{f}}{r_{d}}=\frac{r_{o}-r_{d}}{r_{d}}
$$

i.e., outer finger length $r_{f}$ over compact invasion radius $r_{d}$, during the growth of the invasion patterns for all the experiments. For $\Delta P=10 \mathrm{cmH}_{2} \mathrm{O}$ we generally do not see the signs of a growing compact zone, and $R$ is linearly increasing with $r_{o}$ except for a cutoff when the structure for $C_{g}=0 \%$ becomes large. However, the structure for $C_{g}=0 \%$ is in the capillary fingering regime with active growth everywhere in the medium. Eventually, as the structure grows, central invasions fill up the region close to the inlet and our algorithm captures it as a compact invasion. The other two structures $\left(C_{g}=50\right.$ and $\left.90 \%\right)$ have crossed over to the VF regime and shows no compact invasion behavior. For $\Delta P=30$ and $50 \mathrm{cmH}_{2} \mathrm{O}$ we see a clear transition in the behavior of $R\left(r_{o}\right)$ for decreasing $C_{g}$, where the linearly increasing trend indicating VF has a transition to a behavior where $R$ stabilizes toward a more or less stable value after an initial growth. The transition occurs between $C_{g}=60$ and $70 \%$ for $\Delta P=30 \mathrm{cmH}_{2} \mathrm{O}$, between $C_{g}=70$ and $80 \%$ for $\Delta P=50 \mathrm{cmH}_{2} \mathrm{O}$, and we see that $R$ stabilizes at around $r_{o}=120 \mathrm{~mm}$. The average value of $R\left(r_{o}>120 \mathrm{~mm}\right)$ generally decreases with decreasing $C_{g}$, as well as with increasing $\Delta P$. Interestingly, this behavior indicates a crossover from viscous fingering to the growth of an internal compact invasion with a radius that is proportional to the pattern size and that this proportional size increases with viscosity ratio (and/or 

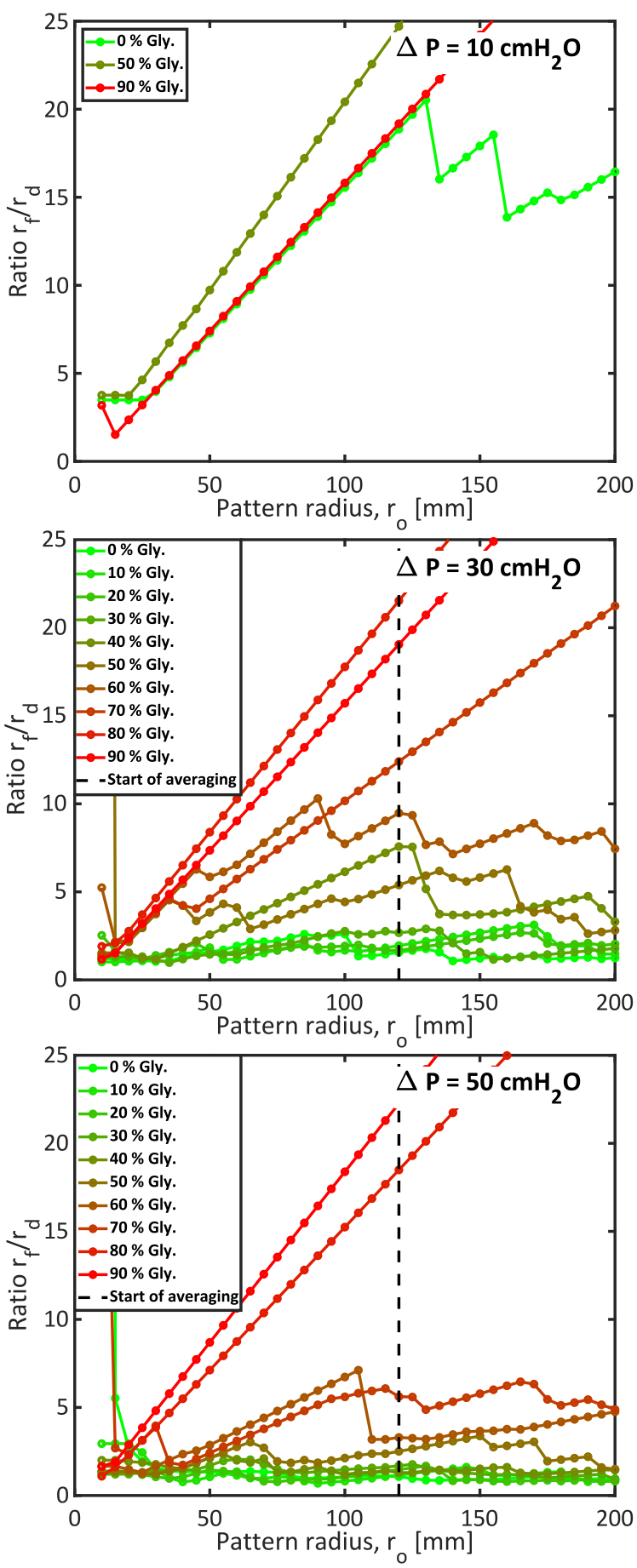

FIG. 7. The ratio $r_{f} / r_{d}$ (outer finger length/compact invasion radius) is plotted as function of the outer radius $r_{o}$ for all the experiments. The line color goes from green to red for increasing defending liquid viscosity. The vertical dashed line in the plots for $\Delta P=30$ and $50 \mathrm{cmH}_{2} \mathrm{O}$ indicates where the invasion pattern stabilizes for compact invasion $\left(r_{o}=120 \mathrm{~mm}\right.$ ), after which we consider the ratio $R$ between the outer fingers and dense invasion to have stabilized (the points to the right of $r_{o}=120 \mathrm{~mm}$ are used to make Fig. 8). 


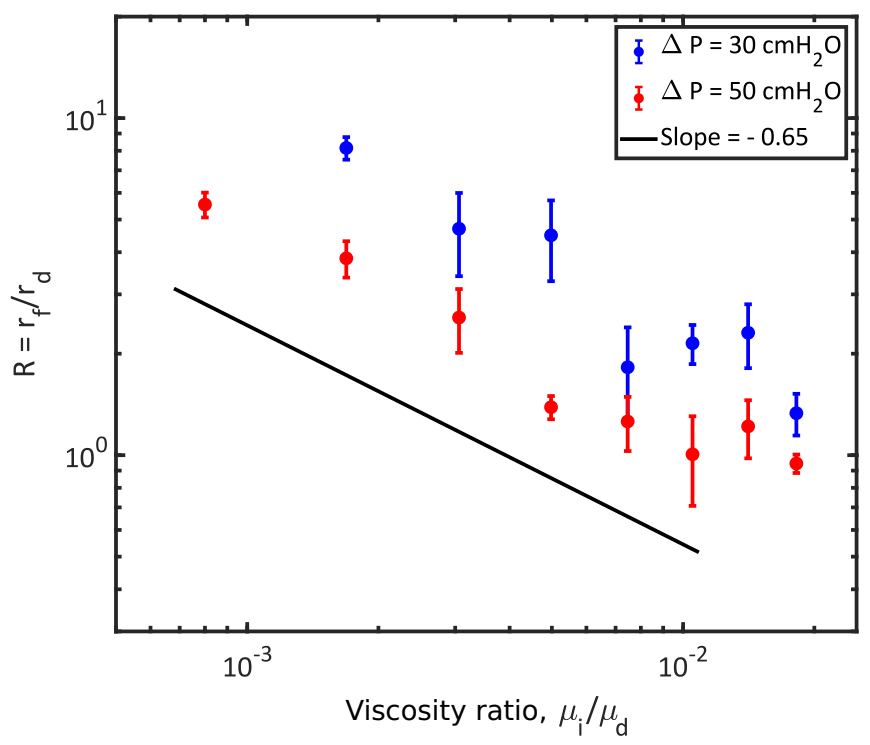

FIG. 8. The ratio $R=r_{f} / r_{d}$ (outer finger length/compact invasion radius) is plotted as function of the viscosity ratio $M=\mu_{i} / \mu_{d}$ between the invading air and the defending liquid. The plot shows the average $R$ as the pattern grows from $r_{o}=120 \mathrm{~mm}$ to $200 \mathrm{~mm}$ for two sets of experiments: one with $\Delta P=30 \mathrm{cmH}_{2} \mathrm{O}$ (blue circles) and the other with $\Delta P=50 \mathrm{cmH}_{2} \mathrm{O}$ (red circles). Only the experiments where compact invasion was observed are included. The error bars indicate the standard deviation of $R$. The black line is a guide to the eye to indicate the scaling of $R$ with $M$.

$\Delta P)$. Animations in the supplementary material show examples of such proportionate growth, see S5 and S6 in Ref. [37].

Figure 8 shows the scaling of $R$ with respect to the viscosity ratio between the air and the defending liquid,

$$
M=\frac{\mu_{i}}{\mu_{d}},
$$

for the experiments where compact invasion was observed (from $0 \%$ to $60 \%$ glycerol for $30 \mathrm{cmH}_{2} \mathrm{O}$ and $0 \%$ to $70 \%$ glycerol for $50 \mathrm{cmH}_{2} \mathrm{O}$ as can be seen in Fig. 7). The filled circles show the average of $R\left(r_{o}\right)$ taken from $r_{o}=120 \mathrm{~mm}$ until breakthrough $\left(r_{o}=200 \mathrm{~mm}\right)$, where the blue color indicates $\Delta P=30 \mathrm{cmH}_{2} \mathrm{O}$ and red color $50 \mathrm{cmH}_{2} \mathrm{O}$. For $M>10^{-3}$ we see a behavior consistent with a power-law decay in $R$ with a similar exponent for both injection pressures. The black line is a guide to the eye placed with an exponent of -0.65 , where \pm 0.05 is a rough estimate for the uncertainty of the exponent value. An increase in overpressure seems to decrease the value of $R(M)$, suggesting that increased injection pressures will move the onset of compact invasion to lower viscosity ratios. In Ref. [30] the authors report that the ratio $R$ scales with $M$ with an exponent of -0.76 for $M \in\left[10^{-3}, 10^{-1}\right]$, which is a similar behavior to what we see in the consistent range of $M \in\left[10^{-3}, 10^{-2}\right]$. In the same article, it is also reported that the structures exhibit proportionate growth in the regime where fingers without tip splitting are observed. It is interesting to note the similarities between our work and the results in Ref. [30], since we have immiscible flow in a porous medium while Ref. [30] studies miscible flow in an empty Hele-Shaw cell.

\section{Pore-pressure evolution}

As described in the methodology, we conducted a few experiments where the pore overpressure $\Delta P_{p}$ was measured relative to the atmosphere at five points along a line radiating out from the 

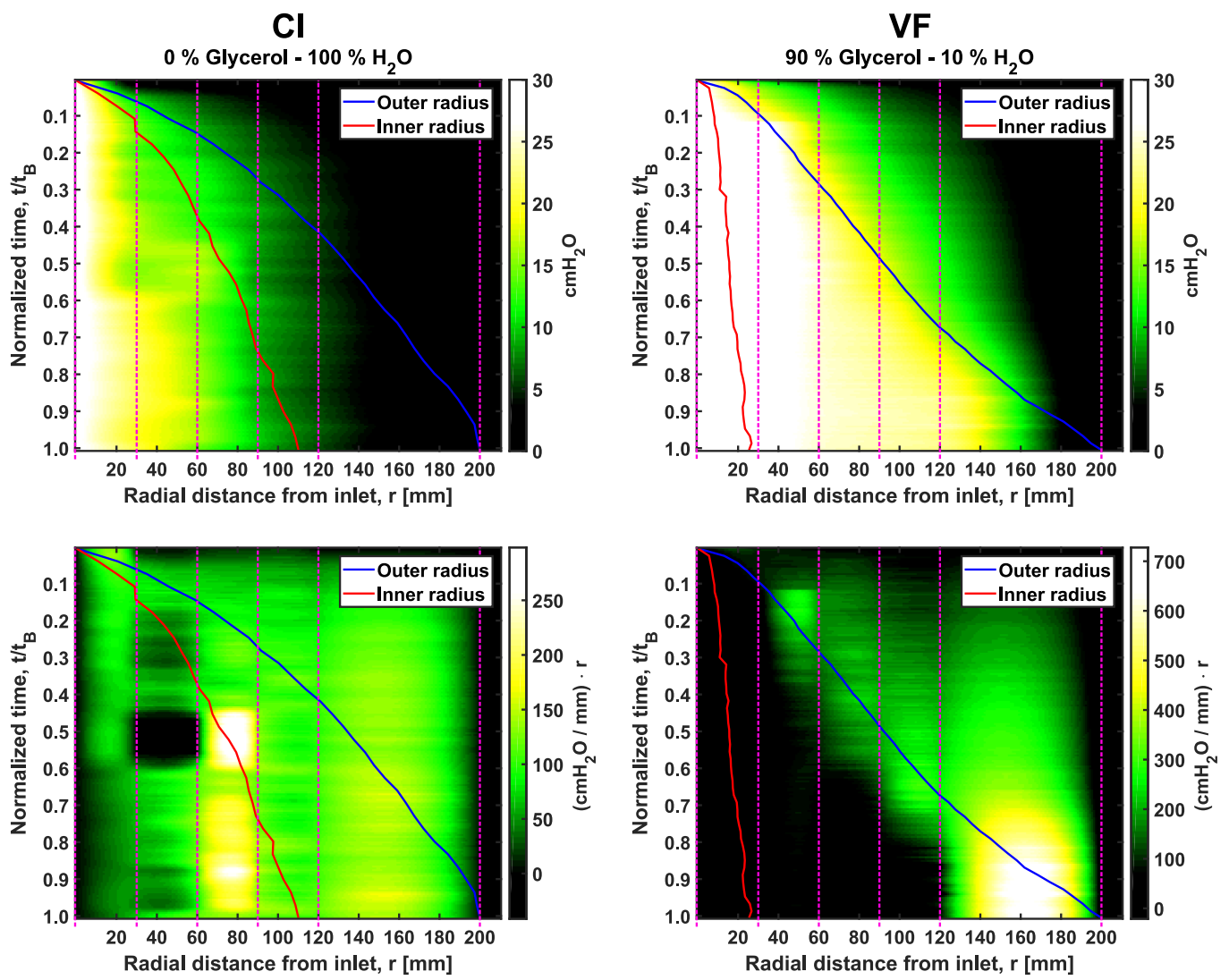

FIG. 9. The two top panels show the evolution of pore overpressure $\Delta P_{p}$ during the experiments with the lowest viscosity (left, 100\% water) and the highest viscosity (right, 90\% glycerol-10\% water) for injection pressure $\Delta P=30 \mathrm{cmH}_{2} \mathrm{O}$. The pressure map as function of radius and time (normalized by the experiment's duration) is given by the colorbar, and the evolution of $r_{d}$ (inner radius) and $r_{o}$ (outer radius) is plotted on top. The vertical magenta dashed lines indicate the radii where we measure or know the pressure, and the pressure map between these points is shown by using shape-preserving cubic spline interpolation. The bottom row of panels visualize the evolution of the radial pressure gradient for the same experiments as in the top row. The gradient maps are multiplied with $r$ for visualization purposes, accounting for $\nabla P \sim 1 / r$ (radial flow). The figure shows that in the CI regime (left), we do not measure a strong screening of pressure in the internal parts of the invasion structure as we measure and expect in the VF regime (right). The presence of an internal pressure drop in the compact invasion regime is a clear distinction from the viscous fingering regime and could be a driving force for central pore invasions even when the invasion front is far ahead.

inlet (green stars in Fig. 1). We use these pressure measurements to visualize the evolution of the pore pressure by creating spatiotemporal pressure maps. These maps are made by stacking the measurements below another as function of time, where each measurement is interpolated in space between the sensors using a shape preserving cubic interpolation (Piecewise Cubic Hermite Interpolating Polynomial, "pchip" in Matlab). Note that we know the outlet overpressure to be $\Delta P_{p}=0$, and we use this as a point in the interpolation.

The evolution of $\Delta P_{p}$ over time is shown for two experiments in Fig. 9, where the left column shows an experiment with pure water (least viscous defending liquid) and the right column shows an experiment with $90 \%$ glycerol (most viscous defending liquid) where both are injected with air at $30 \mathrm{cmH}_{2} \mathrm{O}$ overpressure. In other words, the left column shows the pore-pressure evolution in the compact invasion regime while the right column shows the pore-pressure evolution in the viscous 
fingering regime. Furthermore, the top row of Fig. 9 shows the measured pressure and the bottom row visualizes the pressure gradient in the radial direction from the inlet. Note that the gradient maps are multiplied with $r$, accounting for $\nabla P \sim 1 / r$ (radial flow) for visualization purposes.

For the viscous fingering experiment we see that the pressure field (top right) grows steadily outwards with time and that the pressure field is relatively uniform at a short distance behind the longest fingers (blue line), i.e., a strong screening of the pressure gradient inside the longest finger tips. Note the boundary effect on the interpolation for normalized time $>0.9$ and $r \approx 160$ to $200 \mathrm{~mm}$, where the outlet pressure reduces the visualized pressure due to the long step from sensor 5 to the outlet. As shown in the corresponding visualization of the pressure gradient (bottom right), the strongest pressure gradients follow the outer radius of the invasion pattern (except for the boundary effect for normalized time $>0.9$ ). This behavior is what we expect to see for viscous fingers where the most advanced tips grow on expense of the less advanced ones. On the other hand, we see a clearly different behavior in the compact invasion regime. In the compact invasion regime, the pressure field (top left) grows out into the model initially and then stabilizes toward a configuration where the significant overpressure remains focused within a $100-\mathrm{mm}$ radius. In the corresponding visualization of the pressure gradient (bottom left), we see that it suggests a quite stable $\nabla P \sim 1 / r$ relationship as if there was only a radial single-phase flow, i.e., $\nabla P \cdot r$ is shown as roughly constant with fluctuations. The local fluctuations seen in the pressure gradient close to the inlet are linked to whether there is air or liquid at the pressure sensor: For example, at normalized time $\approx 0.5$ there is air with higher pressure at sensor $3(60 \mathrm{~mm})$ and liquid (with lower pressure) at the neighboring sensors. The important observation in Fig. 9 is that the pressure gradients are not screened behind the longest finger tips in the compact invasion regime.

\section{E. Ganglion dynamics}

As mentioned in Sec. II C, we have employed the flipping matrix concept to visualize and quantify the ganglion dynamics in each experiment. The flipping matrix is computed at breakthrough and it measures the number of times the state of a given pixel in the image has flipped between liquid filled and air filled, see Fig. 3(b). Similarly to what was shown for the invading matrix in Fig. 6, we have computed the activity landscape (3D representation of the flipping matrix) for all experiments and present a selection of the data in the diagram in Fig. 10. A more complete high resolution version of this diagram including all 23 experiments can be found in the supplementary material, see S7 in Ref. [37].

We can immediately see that the bottom line (lowest air pressure) and rightmost column (highest liquid viscosity), are dominated by pixels having activity order 1 (dark blue, the primary drainage plateau mentioned in Sec. II D), i.e., we find very little ganglion activity in this region. The speckles with activity order larger than 1 seem to be concentrated around the region of low viscosities and high invading pressures. This is precisely the zone where the Reynolds number is expected to be higher and inertial effects seem to be more dominant, as mentioned in Sec. III B.

To further analyze the ganglion dynamics, we compute the global measures of ganglion dynamics intensity $\psi_{1}$ and $\psi_{2}$, as explained in Sec. II D. In Fig. 11, we show a contour plot of $\psi_{1}$ and $\psi_{2}$. To produce the plots, we have initially computed $\psi_{1}$ and $\psi_{2}$ for the 23 experiments performed. The experiments are marked by the diamonds in the figure. The two diamonds highlighted in green on each figure correspond to the experiments analyzed in Fig. 9 (we will get back to this point in the following section). Once the value of $\psi_{1}$ and $\psi_{2}$ is computed for all experiments, the value for the other points in the diagram are obtained via a cubic $2 \mathrm{D}$ spline interpolation. This interpolation algorithm produces smooth 3D surfaces for $\psi_{1}$ and $\psi_{2}$, continuous and with continuous derivatives up to the second order.

From the analysis of Fig. 11 we see that both measures $\psi_{1}$ and $\psi_{2}$ produce very similar contour plots, something expected as those measures are expected to be correlated [for porous media without large heterogeneities in permeability (like fractures), as discussed in Sec. II D]. In our case, $\psi_{1}$ varies roughly in the interval $(0,0.5)$ and $\psi_{2}$ in the interval $(0,0.35)$. Both measures indicate that 


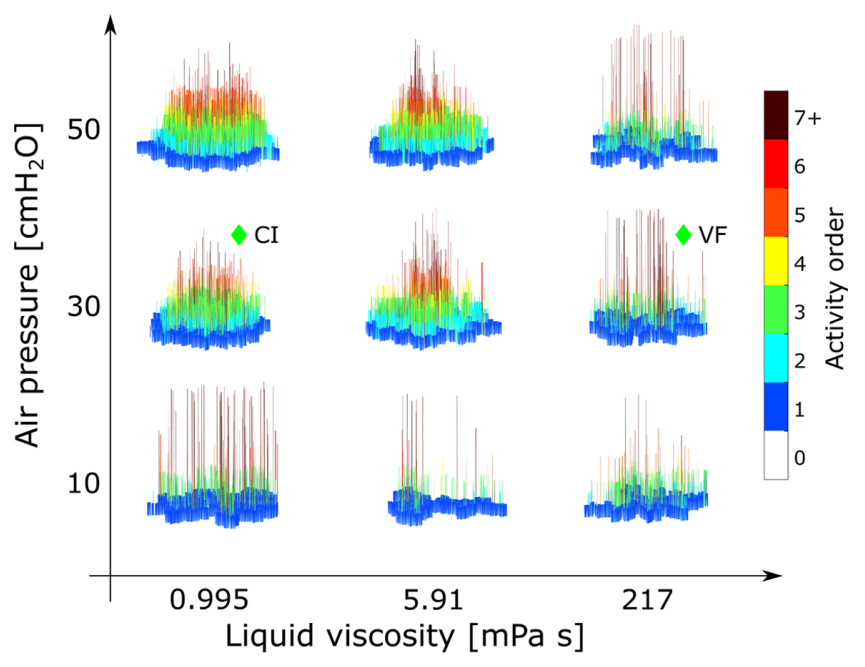

FIG. 10. Activity landscape diagram. The colormap indicates the activity order of a given pixel, i.e., the number of times that pixel has flipped states (see video in supplementary material S2 [37]). Notice that the ganglion dynamics is more intense for experiments with high invading pressure and lower viscosities. The two experiments marked CI and VF correspond to the same experimental conditions from the experiments on the left and right columns of Fig. 9. A complete version of this diagram with all 23 experiments analyzed is found in the supplementary material S7 in Ref. [37].

the ganglion dynamics intensity grows toward the upper left corner (lower viscosities and higher pressures), as we expect from the analysis of the activity landscape diagram in Fig. 10, where we observe more pronounced domes in the experiments toward the upper left corner. The fact that both measures $\psi_{1}$ and $\psi_{2}$ lead to similar conclusions attests in favor of our argument that although $\psi_{1}$ is more prone to errors, the number of pixels that have their activity order miscounted becomes statistically negligible in comparison with the total number of invaded pixels. Although the associated errors in the measurement of $\psi_{1}$ can be safely neglected here, it is important to notice that we expect the measurements for the fastest experiments (corresponding to the lowest viscosity values) to be more prone to such errors. The reason for this is because when the viscosity is reduced, the fluids speed increase, which makes it harder to image all flipping events accurately. If we had access to an even higher acquisition rate, then we would expect the measured values of both $\psi_{1}$ and $\psi_{2}$ to be even higher in the upper left corner of the plots in Fig. 11.

\section{F. Link between the ganglion dynamics and the spatial distribution of the pressure field}

Our results in Fig. 9 have led to an important differentiation between the pressure profiles of the compact invasion and viscous fingering regimes. As mentioned, the screening of the pressure field behind the most advanced finger tip, a defining feature of the viscous fingering regime [4], is completely absent in the experiment that led to the compact invasion structure. In this section we will see that the different pressure profiles carry an immediate impact on the ganglion dynamics behind the main invasion front.

Consider again the ganglion dynamics quantification presented in the activity landscape diagram in Fig. 10. The two experiments at the left and right part of the central line $\left(30 \mathrm{cmH}_{2} \mathrm{O}\right.$ overpressure $)$ correspond respectively to the same experimental conditions present in the experiments in the left and right columns of Fig. 9. They are examples of the CI and VF regime, respectively. We show the labels CI and VF in Fig. 10 to aid visualization. We see that the ganglion dynamics activity is much more intense in the compact invasion case than in the viscous fingering case. This is visually observed by the pronounced dome seen in the experiment marked with CI, a feature that is in 

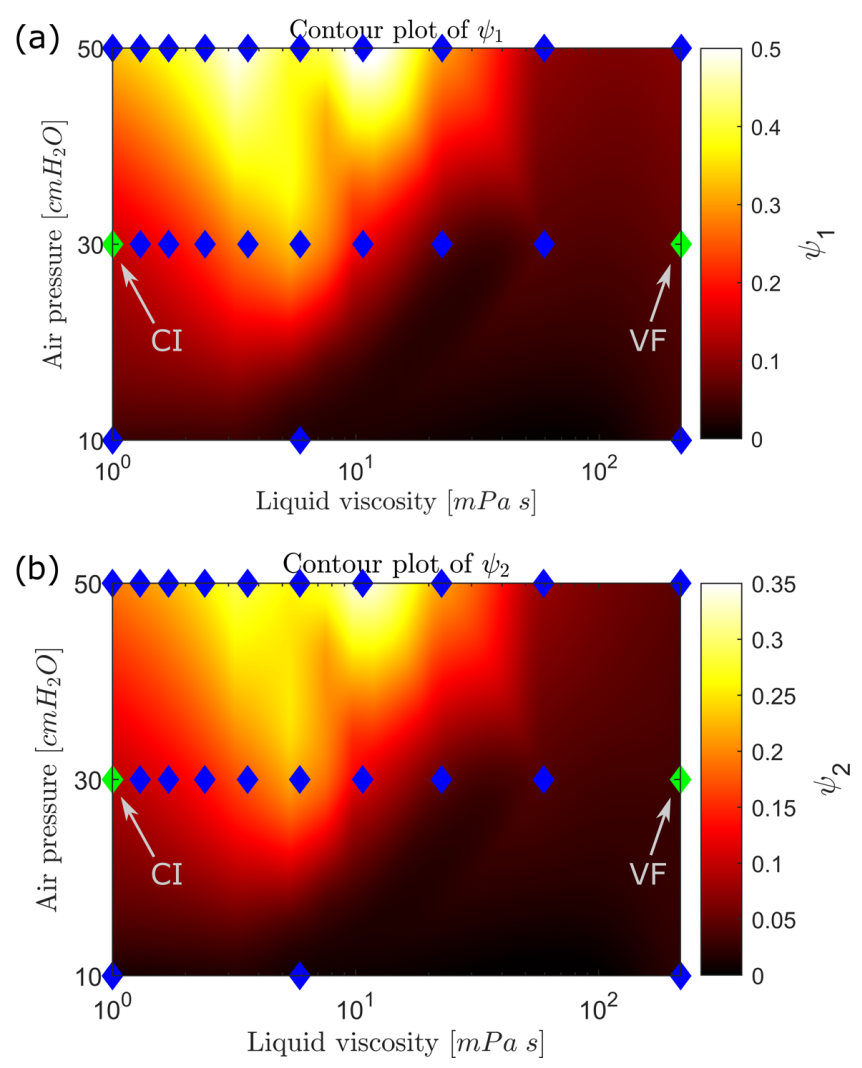

FIG. 11. (a) Contour plot of the ganglion dynamics intensity measure $\psi_{1}$. The ganglion dynamics grows toward the upper left corner of the diagram (lower viscosities and higher pressures), compare with Fig. 6. The diamonds denote the experimental points, where $\psi_{1}$ was measured and the green diamonds highlighted at the extreme sides of the line with pressure $30 \mathrm{cmH}_{2} \mathrm{O}$ correspond to the conditions of the experiment that had the pressure analyzed in Fig. 9 (CI and VF). (b) Similar plot for the ganglion dynamics intensity measure $\psi_{2}$.

complete contradistinction to the observation from the VF experiment, where instead of the dome we see a clear dominance of the primary drainage plateau (activity order $=1$ ). We can also quantify this feature by considering the values of our two measures of global ganglion dynamics intensity $\psi_{1}$ and $\psi_{2}$, as defined in Sec. II D. For the CI experiment we have $\psi_{1}=0.147$ and $\psi_{2}=0.095$ and for the VF experiment we have $\psi_{1}=0.071$ and $\psi_{2}=0.031$ (see points marked by the green diamonds in Fig. 11). Once again we conclude that the ganglion dynamics activity is much more pronounced in the $\mathrm{CI}$ case than in the VF case.

These observations regarding the ganglion dynamics intensity serve as an additional independent verification of the important conclusion derived in Fig. 9, namely that the screening of the pressure field observed in the viscous fingering regime is absent (or at least significantly reduced) in the compact invasion regime. Indeed, while the typical stillness of the front structure behind the most advanced finger tip advocates for the screening of the pressure field, the intense ganglion activity in the CI regime can only be possible if strong pressure gradients are present even in points far away from the most advanced fingers. The intense ganglion dynamics activity is driven by such strong pressure gradients and if the pressure were screened as in the VF case (thus leading to a nearly constant pressure profile behind the invasion front, see right side of Fig. 9), we would not observe such strong ganglion activity. 


\section{DISCUSSION AND CONCLUSIONS}

We have shown experimental evidence for the existence of a rich array of invasion regimes in two-phase flows in porous media, thus extending the classical picture presented in the work of Ref. [11]. In addition to the much studied viscous and capillary fingering regimes, we have seen that we can also have a regime characterized by strong ganglion dynamics as a response to the absence of the viscous fingering pressure screening. This leads to the formation of a much denser invasion profile around the inlet of the model. This compact invasion regime would not be captured by analytical/computational models that do not take into account the effects leading to the strong ganglion activity. In particular, any model in which the invasion state of a given pore can only flip once (like the well-known diffusion-limited aggregation algorithm [28], used to simulate the viscous fingering patterns) by construction would not be able to capture the ganglion activity and the subsequent formation of denser invasion zones. This does not mean this model and other "single state flipping" models (for example the invasion percolation algorithm [14]) are not relevant. Quite the opposite, but, like with all numerical models, one needs to be fully aware of their applicability domains.

We have employed two matricial quantities to study the dynamics of our experiments: the invading matrix, which gives a spatiotemporal map of the primary invasion, and the flipping matrix, used to visualize the ganglion activity. Furthermore, we have defined two global measures of ganglion dynamics intensity defined over the flipping matrix, $\psi_{1}$ and $\psi_{2}$. We have shown how $\psi_{1}$ and $\psi_{2}$ can be employed to quantify the ganglion dynamics and find the region of the experimental parameter space for which the ganglion dynamics is more intense.

We also discovered that when the invading structure becomes large, it appears equal when rescaling with the total structure size. This feature, known as propotionate growth, allowed for the measurement of a well-defined ratio between the length of the external fingers and the length of the compact zone. This ratio was discovered to be dependent on the viscosity ratio $M$, which is consistent with a power-law behavior with an exponent of -0.65 .

Notice that the formation of a compact invasion structure here differs from that observed for example in the work of Lenormand et al. [11]. In that work, the authors showed that in the fast displacement of a less viscous fluid by a more viscous one, a stable compact fluid front would be formed, this front being stabilized by viscous effects in the invading phase. The compact invasion we observe here is not in a stable configuration in this sense, as all our experiments were performed in the scenario that a less viscous fluid is injected into a more viscous one (unstable displacement).

\section{ACKNOWLEDGMENTS}

This work received funding from the Research Council of Norway through its Centres of Excellence funding scheme, Project No. 262644, PoreLab CoE. We thank Eirik G. Flekkøy, Renaud Toussaint, and Alex Hansen for fruitful discussions.

[1] D. A. Weitz, J. P. Stokes, R. C. Ball, and A. P. Kushnick, Dynamic Capillary Pressure in Porous Media: Origin of the Viscous-Fingering Length Scale, Phys. Rev. Lett 59, 2967 (1987).

[2] J.-D. Chen and D. Wilkinson, Pore-Scale Viscous Fingering in Porous Media, Phys. Rev. Lett. 55, 1892 (1985).

[3] K. J. Måløy, J. Feder, and T. Jøssang, Viscous Fingering Fractals in Porous Media, Phys. Rev. Lett. 55, 2688 (1985).

[4] G. Løvoll, Y. Meheust, R. Toussaint, J. Schmittbuhl, and K. J. Måløy, Growth activity during fingering in a porous hele shaw cell, Phys. Rev. E 70, 026301 (2004).

[5] R. Lenormand and C. Zarcone, Invasion Percolation in an Etched Network: Measurement of a Fractal Dimension, Phys. Rev. Lett 54, 2226 (1985). 
[6] N. Martys, M. O. Robins, and M. Cieplak, Scaling relations for interface motion through disordered media: Application to two-dimensional fluid invasion, Phys. Rev. B 44, 12294 (1991).

[7] A. Birovljev, L. Furuberg, J. Feder, T. Jøssang, K. J. Måløy, and A. Aharony, Gravity Invasion Percolation in Two Dimensions: Experiment and Simulation, Phys. Rev. Lett. 67, 584 (1991).

[8] B. Zhao, C. W. Mac Minn, and R. Juanes, Wettability control on multiphase flow in patterned microfluidics, Proc. Natl. Acad. Sci. USA 113, 10251 (2016).

[9] B. B. Mandelbrot, The Fractal Geometry of Nature (W. H. Freeman, San Fransisco, 1982).

[10] J. Feder, Fractals (Plenum, New York, 1988).

[11] R. Lenormand, E. Touboul, and C. Zaarcone, Numerical models and experiments on immiscible displacement in porous media, J. Fluid. Mech. 189, 165 (1988).

[12] P. de Gennes and E. Guyon, Lois generales pour l'injections d'un fluide dans un milieu poreux aleatoire, J. Mech. 17, 403 (1978).

[13] R. Chandler, J. Koplik, K. Lerman, and D. Willemsen, Capillary displacement and percolation in porous media, J. Fluid. Mech. 119, 249 (1982).

[14] D. Wilkinson and J. F. Willemsen, Invasion percolation: A new form of percolation theory, J. Phys A: Math. Gen. 16, 3365 (1983).

[15] M. Moura, E.-A. Fiorentino, K. J. Måløy, G. Schäfer, and R. Toussaint, Impact of sample geometry on the measurement of pressure-saturation curves: Experiments and simulations, Water Resour. Res. 51, 8900 (2015).

[16] M. Moura, K. J. Måløy, E. G. Flekkøy, and R. Toussaint, Verification of a Dynamic Scaling for the Pair Correlation Function During the Slow Drainage of a Porous Medium, Phys. Rev. Lett. 119, 154503 (2017).

[17] P. Saffman and G. Taylor, The penetration of a fluid into a porous medium or Hele-Shaw cell containing a more viscous liquid, Proc. R. Soc. London A 245, 312 (1958).

[18] M. Ferer, C. Ji, G. S. Bromhal, J. Cook, G. Ahmadi, and D. H. Smith, Crossover from capillary fingering to viscous fingering for immiscible unstable flow: Experiment and modeling, Phys. Rev. E 70, 016303 (2004).

[19] E. Aker, K. J. Måløy, A. Hansen, and G. Batrouni, A two-dimensional network simulator for two-phase flow in porous media, Transp. Porous Media 32, 153 (1998).

[20] M. Ferer, G. S. Bromhal, and D. H. Smith, Pore-level modeling of drainage: Crossover from invasion percolation to compact flow, Phys. Rev. E 67, 051601 (2003).

[21] O. I. Frette, K. J. Måløy, J. Schmittbuhl, and A. Hansen, Immiscible displacement of viscosity-matched fluids in two-dimensional porous media, Phys. Rev. E 55, 2969 (1997).

[22] M. Ferer, R. A. Geisbrecht, W. N. Sams, and D. H. Smith, Crossover from fractal to compact growth from simulations of two-phase flow with finite viscosity ratio in two-dimensional porous media, Phys. Rev. A 45, R6973 (1992).

[23] M. Ferer, W. N. Sams, R. A. Geisbrecht, and D. H. Smith, Crossover from fractal to compact growth from simulations of two-phase flow with finite viscosity ratio in two-dimensional porous media, Phys. Rev. E 47, 2713 (1993).

[24] R. M. Brady and R. C. Ball, Fractal growth of copper electrodeposits, Nature (London) 309, 225 (1984).

[25] M. Matsushita, M. Sano, Y. Hayakawa, H. Honjo, and Y. Sawada, Fractal Structures of Zinc Metal Leaves Grown by Electrodeposition, Phys. Rev. Lett 53, 286 (1984).

[26] L. Niemeyer, L. Pietronero, and H. J. Wiesmann, Fractal Dimension of Dielectric Breakdown, Phys. Rev. Lett 52, 1033 (1984).

[27] G. Daccord, Chemical Dissolution of a Porous Medium by a Reactive Fluid, Phys. Rev. Lett 58, 479 (1987).

[28] T. A. Witten and L. M. Sander, Diffusion Limited Aggregation, a Kinetic Critical Phenomenon, Phys. Rev. Lett 47, 1400 (1981).

[29] L. Paterson, Diffusion-Limited Aggregation and Two-Fluid Displacement in Porous Media, Phys. Rev. Lett 52, 1621 (1984).

[30] I. Bischofberger, R. Ramachandran, and S. R. Nagel, Fingering versus stability in the limit of zero interfacial tension, Nat. Commun 5, 5265 (2014). 
[31] A. C. Payatakes, K. M. Ng, and R. W. Flumerfelt, Oil ganglion dynamics during immiscible displacement: Model formulation, AIChE J. 26, 430 (1980).

[32] K. T. Tallakstad, H. A. Knudsen, T. Ramstad, G. Løvoll, K. J. Måløy, R. Toussaint, and E. G. Flekkøy, Steady-State Two-Phase Flow in Porous Media: Statistics and Transport Properties, Phys. Rev. Lett. 102, 074502 (2009).

[33] M. Erpelding, S. Sinha, K. T. Tallakstad, A. Hansen, E. G. Flekkøy, and K. J. Måløy, History independence of steady state in simultaneous two-phase flow through two-dimensional porous media, Phys. Rev. E 88, 053004 (2013).

[34] T. Ramstad and A. Hansen, Cluster evolution in steady-state two-phase flow in porous media, Phys. Rev. E 73, 026306 (2006).

[35] N.-S. Cheng, Formula for the viscosity of a glycerol-water mixture, Industr. Eng. Chem. Res. 47, 3285, (2008).

[36] J. Rumble, CRC Handbook of Chemistry and Physics: A Ready-reference Book of Chemical and Physical Data (CRC Press, Boca Raton, FL, 2019).

[37] See Supplemental Material at http://link.aps.org/supplemental/10.1103/PhysRevFluids.7.013901 for videos, supporting figures, and animations.

[38] U. Oxaal, M. Murat, F. Boger, A. Aharony, J. Feder, and T. Jøssang, Viscous fingering on percolation clusters, Nature (London) 329, 32 (1987).

[39] R. Lenormand, Flow through porous media: Limits of fractal pattern, Proc. R. Soc. Lond. A 423, 159 (1989).

[40] M. Moura, K. J. Måøy, E. G. Flekkøy, and R. Toussaint, Intermittent dynamics of slow drainage experiments in porous media: Characterization under different boundary conditions, Front. Phys. 7, 217 (2020).

[41] M. Moura, E. G. Flekkøy, K. J. Måløy, G. Schäfer, and R. Toussaint, Connectivity enhancement due to film flow in porous media, Phys. Rev. Fluids 4, 094102 (2019).

[42] K. J. Måløy, F. Boger, J. Feder, T. Jøssang, and P. Meakin, Dynamics of viscous-fingering fractals in porous media, Phys. Rev. A 36, 318 (1987).

[43] R. Toussaint, G. Løvoll, Y. Méheust, K. J. Måløy, and J. Schmittbuhl, Influence of pore-scale disorder on viscous fingering during drainage, Europhys. Lett. 71, 583 (2005).

[44] G. Løvoll, M. Jankov, K. Måløy, R. Toussaint, J. Schmittbuhl, G. Schäfer, and Y. Méheust, Influence of viscous fingering on dynamic saturation-pressure curves in porous media, Transp. Porous Media 86, 305 (2011). 